\title{
Wnt7a predicts poor prognosis, and contributes to growth and metastasis in tongue squamous cell carcinoma
}

\author{
BO JIA $^{1 *}$, XIAOLING QIU ${ }^{1 *}$, HONGXING CHU ${ }^{1}$, XIANG SUN $^{1}$, SHUAIMEI XU ${ }^{2}$, \\ $\mathrm{XINYUAN} \mathrm{ZHAO}^{2}$ and JIANJIANG ZHAO ${ }^{1}$ \\ Departments of ${ }^{1}$ Oral Surgery and ${ }^{2}$ Endodontics, Stomatological Hospital, Southern Medical University, \\ Guangzhou, Guangdong 510280, P.R. China
}

Received May 10, 2018; Accepted September 3, 2018

DOI: $10.3892 /$ or.2019.6974

\begin{abstract}
Regional and distant metastases are the principal reasons underlying the high mortality rate associated with tongue squamous cell carcinoma (TSCC); however, the precise molecular mechanisms involved in tongue tumorigenesis remain unknown. The present study aimed to determine the expression and mechanism of regulation of Wnt7a in the growth and metastasis of TSCC. Wnt7a mRNA and protein expression levels were examined in TSCC tissues using reverse transcription-quantitative polymerase chain reaction and immunohistochemical staining. A loss-of-function assay was performed in TSCC cell lines using Wnt7a small interfering RNA or short hairpin RNA, after which, cell proliferation, migration and invasion were analyzed using Cell Counting Kit-8, tumorigenicity and Transwell assays, respectively. Epithelial-mesenchymal transition (EMT)-associated proteins were detected by western blotting. The mRNA and protein expression levels of Wnt7a were significantly upregulated in cancer tissues compared with in the adjacent non-cancerous tissues. Clinical analysis indicated that Wnt7a expression was associated with $\mathrm{T}$ classification, lymph node metastasis and pathological differentiation, and high Wnt7a expression predicted a short recurrence-free survival for patients with TSCC. Silencing Wnt7a expression suppressed cell proliferation, migration and invasion, and reversed the EMT phenotype in TSCC cell lines. The present study revealed that Wnt7a may be upregulated in TSCC, where it may participate in modulating cell proliferation, migration, invasion and the EMT of TSCC. Therefore, Wnt7a should be considered a novel
\end{abstract}

Correspondence to: Professor Jianjiang Zhao, Department of Oral Surgery, Stomatological Hospital, Southern Medical University, 366 South Jiangnan Avenue, Guangzhou, Guangdong 510280, P.R. China

E-mail: zhaojianjiangzjj@163.com

${ }^{*}$ Contributed equally

Key words: Wnt7a, tongue squamous cell carcinoma, metastasis, recurrence, epithelial-mesenchymal transition oncogene, and a potential prognostic and therapeutic target for patients with TSCC.

\section{Introduction}

Tongue squamous cell carcinoma (TSCC) is one of the most common types of head and neck cancer (HNC) $(1,2) ;>3,000$ people worldwide are diagnosed with TSCC annually and its accounts for $24 \%$ of all HNC cases (3). Although many patients are identified at the earlier stages of the disease, the 5-year survival rate for advanced-stage TSCC is only $\sim 50 \%$, due to high invasiveness and metastasis (4). In addition, no effective drugs are currently available for the treatment and control of regional and distant metastases of TSCC. Therefore, screening novel methods for the diagnosis, prognosis and treatment of TSCC may result in a significant clinical advancement.

TSCC is caused by the interaction of numerous genes, the increased expression of proto-oncogenes and inhibition of tumor suppressor genes. Furthermore, cell signaling pathways, such as p53 (5), Ras (6), mitogen-activated protein kinase, Janus kinase/signal transducer and activator of transcription (7), Wnt and hedgehog (8) signaling pathways serve important roles in the development of TSCC. The Wnt protein family is a group of 19 secreted glycoproteins (9); Wnt proteins transmit signals to cells through $\geq 15$ receptors or collaborative receptors, thus resulting in regulation of the differentiation, apoptosis and proliferation of epithelial cells $(10,11)$. Postoperative metastasis leads to unfavorable outcomes in patients with TSCC, and epithelial-mesenchymal transition (EMT) is considered the fundamental event of cancer metastasis and recurrence $(12,13)$. It is well known that EMT is regulated by a series of molecular factors, including Wnt signaling-related genes (14). Wnt7a is a member of the Wnt family, which has not yet been studied in TSCC. $\beta$-catenin is a core transducer in the canonical Wnt signaling pathway and is an EMT-associated marker (15). Previous studies have indicated the important role of $\beta$-catenin in EMT, thus bridging a crosstalk between the Wnt pathway and EMT $(16,17)$. In addition, the positive correlation between $\mathrm{Wnt} 7 \mathrm{a}$ and the $\mathrm{Wnt} / \beta$-catenin pathway has been discussed previously (18); therefore, it is of great value to explore the role of Wnt7a in the EMT process during TSCC progression. The present study focused on Wnt7a and the Wnt pathway; however, research regarding the interaction 
between Wnt7a and other pathways may be conducted in the future.

Wnt7a has been reported to be highly associated with the progress of cell growth through regulating transforming growth factor (TGF) $-\beta$ receptor and activating the cancer-associated fibroblast phenotype (19). Wnt7a has been reported to be overexpressed in colorectal, pancreatic, gastric, breast and ovarian carcinoma (20). Conversely, in non-small cell lung carcinoma, Wnt7a may function as a tumor suppressor gene (21). In addition, the Wnt7a gene has been reported to be downregulated by high-frequency hypermethylation in pancreatic carcinoma (22). The overexpression of Wnt7a has been demonstrated to enhance the invasiveness and metastasis of ovarian cancer cells, possibly through the Frizzled receptor (23). These results suggested that Wnt7a may regulate the proliferation and adhesion of cancer cells; however, its role in tumor cell proliferation and migration may be dependent on tumor type. However, the clinical significance of Wnt7a expression in TSCC has not yet been revealed. Therefore, it is of great significance to determine the expression levels of Wnt7a in TSCC tissues, and to assess its clinical implications. Furthermore, the mechanism underlying the regulatory effects of Wnt7a on cell proliferation, metastasis and EMT in TSCC was investigated.

\section{Materials and methods}

Tissue samples. Two independent cohorts, comprising 109 patients with TSCC who were diagnosed, treated and followed up at the Stomatological Hospital, Southern Medical University (Guangzhou, China) between November 2010 and May 2014, were recruited to the present study. All patients provided informed consent for the use of their tissues and clinical data. Specimens, including paired cancerous and non-cancerous tissues, were obtained following surgical resection. Samples were immediately frozen using liquid nitrogen after resection and were stored at $-80^{\circ} \mathrm{C}$ prior to RNA extraction (cohort $1, \mathrm{n}=48$ ), or were fixed in $4 \%$ paraformaldehyde at room temperature for 24-48 $\mathrm{h}$ and embedded in paraffin for immunohistochemistry (IHC; cohort 2, n=61). All patients were initially diagnosed with tongue cancer and did not receive any other treatment prior to surgery. The present study was approved by the Independent Ethics Committee of the Stomatological Hospital, Southern Medical University (approval no. [2018]05).

Cell culture. The CAL-27, SCC-15 and SCC-9 human TSCC cell lines were obtained from the American Type Culture Collection (Manassas, VA, USA). HSC-4 was purchased from the Human Science Research Resources Bank of the Japan Health Sciences Foundation (Tokyo, Japan). Human oral keratinocytes were purchased from ScienCell Research Laboratories, Inc. (San Diego, CA, USA). CAL-27 cells were cultured in Dulbecco's modified Eagle's medium (DMEM; Gibco; Thermo Fisher Scientific, Inc., Waltham, MA, USA), HSC-4 cells were cultured in Eagle's Minimum Essential Medium (Gibco; Thermo Fisher Scientific, Inc.), and SCC-15 and SCC-9 cells were maintained in a 1:1 mixture of DMEM and Ham's F12 medium (Gibco; Thermo Fisher Scientific, Inc.). For complete growth medium, fetal bovine serum (FBS;
Gibco; Thermo Fisher Scientific, Inc.) was added to the media at a final concentration of $10 \%$. The cells were maintained at $37^{\circ} \mathrm{C}$ in a humidified atmosphere containing $5 \% \mathrm{CO}_{2}$.

RNA extraction and reverse transcription-quantitative polymerase chain reaction $(R T-q P C R)$. Total RNA was extracted from tissues and cell lines using RNAiso Plus reagent (Takara Bio, Inc., Otsu, Japan), followed by RT using PrimeScript First-Strand cDNA Synthesis kit (Takara Bio, Inc.), according to the manufacturer's protocols. The mRNA expression levels of Wnt7a were evaluated by qPCR using a SYBR $^{\circledR}$ Premix Ex Taq ${ }^{\mathrm{TM}}$ kit (cat. no. RR420A; Takara Bio, Inc.) on the StepOnePlus Real-Time PCR system (Applied Biosystems; Thermo Fisher Scientific, Inc.). The RT-qPCR primers used were as follows: Wnt7a, forward 5'-CCAGCA AAAACCTTGAGCCC-3', reverse 5'-CCTTGTTCAGGT AGGCAGCA-3'; and $\beta$-actin, forward 5'-CGTCACCAACTG GGACGACA-3' and reverse 5'-CTTCTCGCGGTTGGCCTT GG-3'. $\beta$-actin was employed as an endogenous control. Target and endogenous control genes were amplified in triplicate and the relative mRNA expression levels of Wnt7a for each sample were calculated using the $2^{-\Delta \Delta \mathrm{Cq}}$ method (24).

IHC. Paraffin-embedded sections $(4 \mu \mathrm{m})$ were cut and mounted onto slides, after which, paraffin was removed with xylene and the sections were rehydrated in an alcohol gradient. Subsequently, the sections were treated with citrate buffer for antigen retrieval in a microwave oven for $10 \mathrm{~min}$ at $95^{\circ} \mathrm{C}$ and were then cooled to room temperature. The sections were then immersed in $3 \% \mathrm{H}_{2} \mathrm{O}_{2}$ for $15-20 \mathrm{~min}$ at room temperature, blocked with $5 \%$ bovine serum albumin (EMD Millipore, Billerica, MA, USA) solution for $30 \mathrm{~min}$ at room temperature, and incubated with the Wnt7a primary antibody (cat. no. 10605-1-AP; 1:200; ProteinTech Group, Inc., Chicago, IL, USA) at $4^{\circ} \mathrm{C}$ overnight. The next day, sections were incubated with HRP-conjugated goat anti-rabbit IgG (cat. no. TA130023; OriGene Technologies, Inc., Beijing, China) for $15 \mathrm{~min}$ at room temperature to detect primary antibody. Finally, the slides were incubated with DAB (cat. no. PV-6000; ZSGB-BIO; OriGene Technologies, Inc.) and were observed under an upright light microscope.

Two individual pathologists evaluated positive staining of Wnt7a in TSCC tissues. Cytoplasmic brown staining in tumor cells was considered positive and was scored based on the following criteria: i) Staining intensity: 0, negative Wnt7a staining; 1, weak staining; 2, moderate staining; and 3, strong staining. ii) Percentage of stained tumor cells was categorized into four classes, 0 , negative; $1, \leq 25 \% ; 2,26-50 \% ; 3,51-74 \%$; and $4, \geq 76 \%$. Finally, Wnt7a protein expression was scored semi-quantitatively by multiplying the aforementioned scores.

Gene silencing. When cells reached a confluence of $\sim 70 \%$, small interfering (si)RNAs (Sangon Biotech Co., Ltd., Shanghai, China) targeting Wnt7a mRNA (siWnt7a-1: 5'-AAAUUGUUA AAUAUUGCUGUG-3'; siWnt7a-2: 5'-UUAAUAUUAUUU UUAUCAGAA-3') were transfected at a concentration of $50 \mathrm{nmol} / \mathrm{l}$ to induce transient knockdown of Wnt7a expression using Lipofectamine ${ }^{\circledR} 3000$ transfection reagent (Invitrogen; Thermo Fisher Scientific, Inc.) according to the manufacturer's protocol. The cells were collected for subsequent experiments 
after 48-72 $\mathrm{h}$ incubation. The negative control (NC) sequence was as follows: 5'-UUCUCCGAACGUGUCACGUTT-3'.

For the construction of stable Wnt7a-knockdown cell models, CAL-27 cells underwent lentivirus-mediated infection with short hairpin (sh)RNA (Sangon Biotech Co., Ltd.) targeting Wnt7a mRNA (5'-AAAUUGUUAAAUAUU GCUGUG-3') or the NC sequence (5'-UUCUCCGAACGU GUCACGUTT-3') at a multiplicity of infection (MOI) of 20, according to the manufacturer's protocol. After $8 \mathrm{~h}$ culture, the lentivirus-containing culture medium was replaced with fresh medium. The efficiency of lentivirus-mediated RNA interference was determined by RT-qPCR and western blotting.

Cell proliferation assay. Cell proliferation was measured using the Cell Counting Kit-8 (CCK-8) (Dojindo Molecular Laboratories, Inc., Kumamoto, Japan). CAL-27 and SCC-15 cells were collected, resuspended and seeded onto 96-well plates, at a density of $3 \times 10^{3}$ cells/well. After adherence, cells were transfected with Wnt7a siRNA or NC siRNA. After a further incubation for $0,24,48,72$ and $96 \mathrm{~h}, 10 \mu \mathrm{l} \mathrm{CCK}-8$ reagent was added to each well, and absorbance was measured at $450 \mathrm{~nm}$ using a microplate reader (SpectraMax Plus 384; Molecular Devices, LLC, Sunnyvale, CA, USA) after $2 \mathrm{~h}$ incubation at $37^{\circ} \mathrm{C}$.

Tumorigenicity assay. BALB/c male nude mice (specific pathogen-free grade; weight, 14-16 g; age, 3-4 weeks) were purchased from the Laboratory Animal Center of Southern Medical University. Lentiviral-mediated stable Wnt7a-knockdown CAL-27 cells and NC cells were subcutaneously injected into the flanks of nude mice $(n=5$ in each group). Subsequently, the mice were maintained in a specific pathogen-free grade lab, under the following conditions: Controlled temperature, $23 \pm 2^{\circ} \mathrm{C}$; humidity, $40-70 \%$; 12 -h light/dark cycle) at the Laboratory Animal Center of Nanfang Hospital, Southern Medical University (Guangzhou, China) with ad libitum access to food and water for 4 weeks. The volume of xenograft tumors was monitored every 3 days by measuring the length and width (Volume = length $\mathrm{x}$ width $\mathrm{x}$ width $\mathrm{x} \pi / 6$ ). The animal study was conducted in accordance with the Institutional Animal Care and Use Committee (IACUC) guidelines, and was approved by Experimental Animal Ethics Committee of Southern Medical University (approval no. L2016113).

Transwell migration and invasion assays. For the migration assay, a total of $5 \times 10^{4}$ CAL-27 or SCC- 15 cells transfected with Wnt7a siRNA or NC siRNA were collected and resuspended in $200 \mu \mathrm{l}$ serum-free medium. The cells were added to the upper chamber of a Transwell insert (Corning Incorporated, Corning, NY, USA) placed in a 24-well plate, whereas $500 \mu \mathrm{l}$ complete medium containing $10 \%$ FBS was added to the lower chamber. After $24 \mathrm{~h}$ culture, cells on the upper surface of the filter were removed and cells on the lower surface were stained with $0.1 \%$ crystal violet for $20 \mathrm{~min}$ at room temperature. Cells that migrated through the filters were counted and images were captured in five random fields at a magnification of x400 using an inverted light microscope. For the invasion assay, the chambers were pre-coated with Matrigel matrix (Corning Incorporated) diluted with serum-free medium in a ratio of 1:8.
Western blotting. Cells were lysed in radioimmunoprecipitation assay lysis buffer (Beyotime Institute of Biotechnology, Shanghai, China) supplemented with $1 \%$ protease inhibitors and phenylmethylsulfonyl fluoride. Subsequently, after determining the concentration of samples using a bicinchoninic acid protein assay kit (cat. no. K3000; Shanghai Bocai Biotechnology Co., Ltd., Shanghai, China), $30 \mu \mathrm{g}$ protein was separated by $10 \%$ SDS-PAGE and transferred onto polyvinylidene difluoride membranes (EMD Millipore). After blocking non-specific antigens with 5\% skimmed milk for $1 \mathrm{~h}$ at room temperature, membranes were incubated with primary antibodies at $4^{\circ} \mathrm{C}$ overnight. Subsequently, the membranes were washed and incubated with HRP-conjugated goat anti-rabbit secondary antibody (cat. no. D110058; 1:10,000; BBI Life Sciences, Shanghai, China) for $1 \mathrm{~h}$ at room temperature. Finally, signals were visualized using the enhanced chemiluminescence detection system (Hangzhou Fude Biological Technology Co., Ltd., Hangzhou, China). The primary antibodies used were as follows: Anti-Wnt7a rabbit polyclonal antibody (cat. no. 10605-1-AP; 1:1,000; ProteinTech Group, Inc.), anti-E-cadherin rabbit polyclonal antibody (cat. no. 20874-1-AP; 1:1,000; ProteinTech Group, Inc.), anti-N-cadherin rabbit polyclonal antibody (cat. no. 22018-1-AP; 1:1,000; ProteinTech Group, Inc.), anti-Vimentin rabbit monoclonal antibody (cat. no. 5741T; 1:1,000; Cell Signaling Technology, Inc., Danvers, MA, USA), anti- $\beta$-catenin rabbit monoclonal antibody (cat. no. 8480T; 1:1,000; Cell Signaling Technology, Inc.), anti-Snail rabbit monoclonal antibody (cat. no. 3879T; 1:1,000; Cell Signaling Technology, Inc.) and anti- $\beta$-actin rabbit polyclonal antibody (20536-1-AP; 1:10,000; ProteinTech Group, Inc.). $\beta$-actin was used as an endogenous protein for normalization.

Statistical analysis. Statistical analysis was conducted using IBM SPSS Statistics 20.0 (IBM Corporation, Armonk, NY, USA). Data from triplicate experiments are presented as the means \pm standard error of the mean. The associations between Wnt7a mRNA/protein expression and clinicopathological parameters were assessed using the $\chi^{2}$ test. Student's t-test was used to analyze the differences between two groups. One-way analysis of variance followed by least significant difference test, or Kruskal-Wallis test, was used to compare multiple groups. Survival data were evaluated using the Kaplan-Meier method and log-rank test. $\mathrm{P}<0.05$ was considered to indicate a statistically significant difference.

\section{Results}

Upregulation of Wnt7a in human TSCC tissues. Wnt7a expression was detected in the two cohorts of patients with TSCC, which comprised 109 pairs of TSCC specimens and adjacent non-cancerous tissues, using RT-qPCR and IHC. The present study aimed to determine the role of Wnt7a in TSCC. In cohort 1 , upregulation of Wnt7a mRNA was observed in TSCC tissues in $72.9 \%$ (35/48) of patients (Fig. 1A). The mRNA expression levels of Wnt7a were significantly increased in TSCC tissues compared with in the corresponding adjacent normal tissues (Fig, 1B). Furthermore, IHC staining revealed that Wnt7a protein, which is primarily located in the cytoplasm, was significantly overexpressed in TSCC tissues compared 


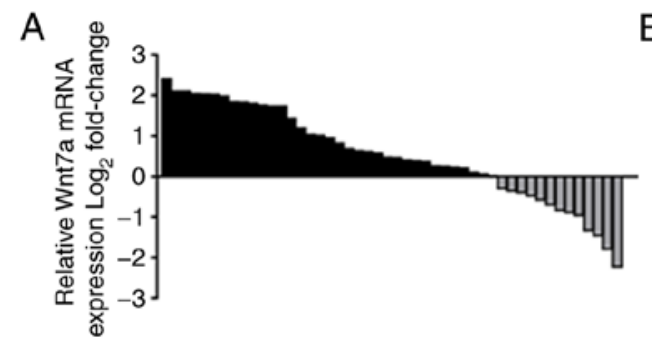

D

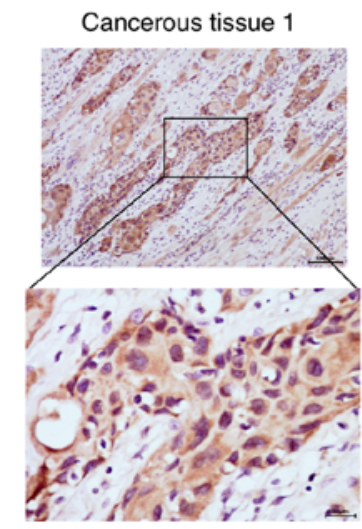

E

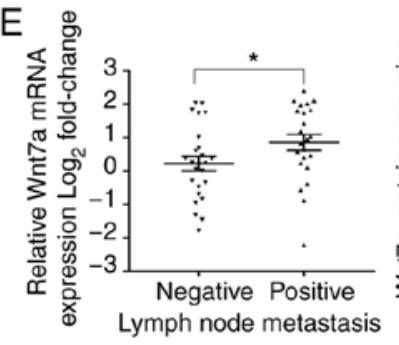

G
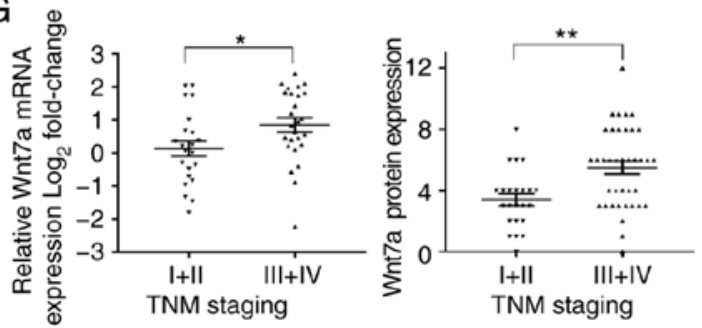

B

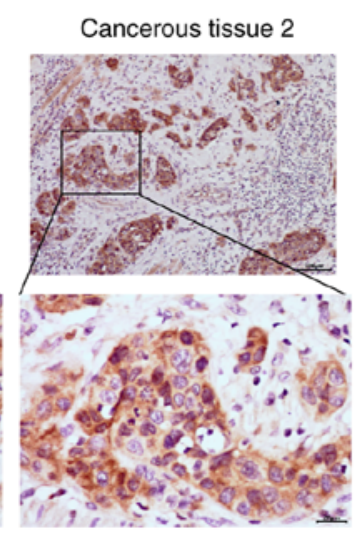

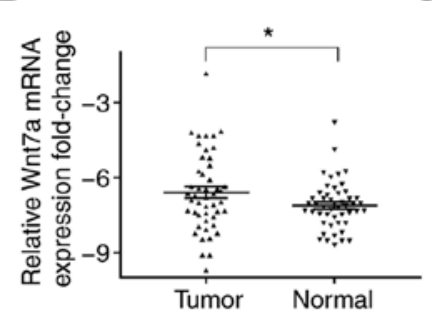

Adjacent normal tissue

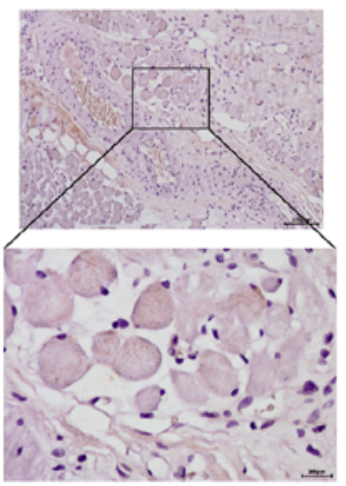

F

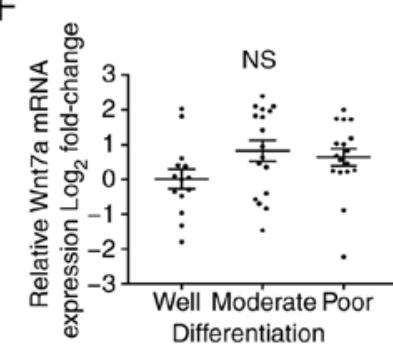

$\mathrm{H}$

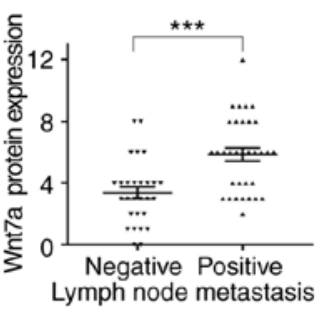

C

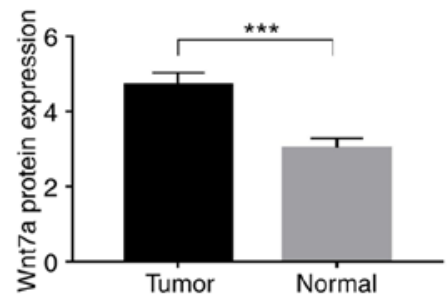

Adjacent normal tissue 2

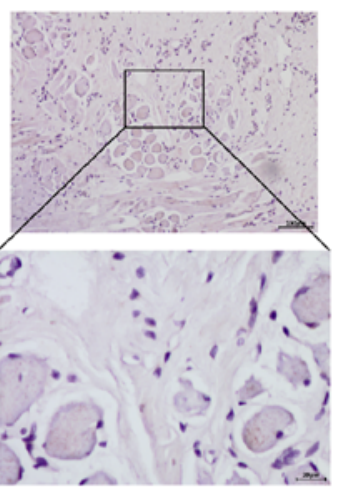

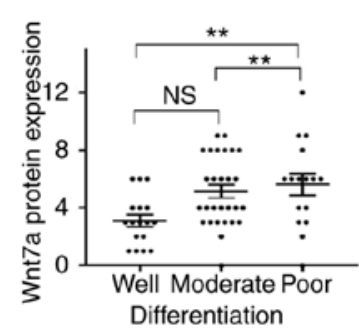
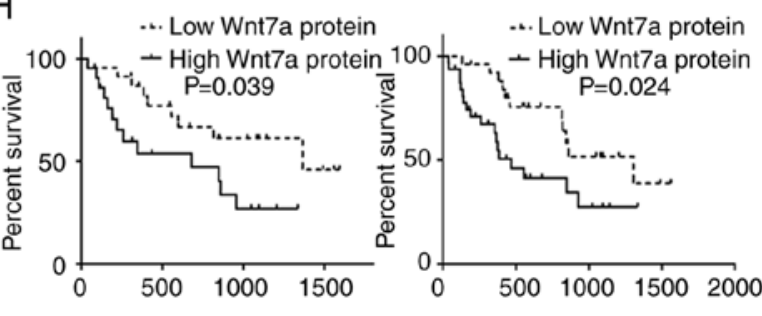

Figure 1. Wnt7a mRNA and protein expression in patients with TSCC, and the association between Wnt7a expression and recurrence-free survival. (A) Alterations in Wnt7a expression in TSCC tissues compared with in adjacent normal tissues. (B) Wnt7a mRNA was upregulated in TSCC tissues compared with that in matching non-cancerous tissues (Student's t-test). (C) Wnt7a protein was upregulated in TSCC tissues compared with that in the matching non-cancerous tissues (Student's t-test). (D) Immunohistochemical staining exhibited Wnt7a protein expression in TSCC and adjacent normal tissues (magnification: Upper panel, $x 100$, scale bars $=100 \mu \mathrm{m}$; lower panel, $\mathrm{x} 400$, scale bars $=20 \mu \mathrm{m}$ ). (E) Wnt7a mRNA and protein expression was higher in tissues obtained from patients with TSCC with lymph node metastasis than those without lymph node metastasis (Student's t-test). (F) Wnt7a protein expression was higher in ESCC tissues with poor differentiation than those with well or moderate differentiation (one-way analysis of variance and least significant difference test); however, no significant difference was observed in Wnt7a mRNA expression between groups (Kruskal-Wallis test). (G) Wnt7a mRNA and protein expression were higher in tissues obtained from patients with advanced TSCC than those with early stage TSCC (Student's t-test). (H) Kaplan-Meier analysis demonstrated that patients with higher Wnt7a mRNA and protein expression had a shorter recurrence-free survival compared with those with lower Wnt7a expression (log-rank test). ${ }^{*} \mathrm{P}<0.05,{ }^{* *} \mathrm{P}<0.01,{ }^{* * *} \mathrm{P}<0.001$. NS, not significant; TSCC, tongue squamous cell carcinoma.

with in matching adjacent normal tissues (Fig. 1C and D). These results suggested that Wnt7a was upregulated in TSCC tissues and may exert a role in carcinogenesis and its progression.

Association between Wnt7a expression and clinicopathological parameters in patients with TSCC. The association between Wnt7a expression and clinicopathological parameters was analyzed to assess the effects of Wnt7a on TSCC. In cohort 1, the patients were categorized into low or high expression groups according to the median value. Wnt7a mRNA expression was strongly associated with $\mathrm{T}$ classification $(\mathrm{P}=0.039)$, lymph node metastasis $(\mathrm{P}=0.020)$, pathological differentiation $(\mathrm{P}=0.037)$ and clinical stage $(\mathrm{P}=0.020$; Table $\mathrm{I})$. In cohort 2 , TSCC tissues with a staining index of $>4$ were defined as the high expression group, whereas those with a staining index $\leq 4$ were defined as the low expression group. Results demonstrated that Wnt7a protein expression was positively associated with $\mathrm{T}$ classification $(\mathrm{P}=0.033)$, lymph node metastasis $(\mathrm{P}=0.001)$, pathological differentiation $(\mathrm{P}<0.0001)$, and clinical stage $(\mathrm{P}=0.002$; Table II). Further analyses revealed that $\mathrm{Wnt} 7 \mathrm{a}$ protein expression was 
Table I. Association between Wnt7a mRNA expression and clinicopathological parameters in patients with tongue squamous cell carcinoma $(n=48)$.

\begin{tabular}{|c|c|c|c|}
\hline \multirow[b]{2}{*}{ Clinicopathological parameters } & \multicolumn{2}{|c|}{ Wnt7a mRNA } & \multirow[b]{2}{*}{ P-value } \\
\hline & Low $(n=24)(\%)$ & $\operatorname{High}(\mathrm{n}=24)(\%)$ & \\
\hline Gender & & & 0.365 \\
\hline Male & $14(45.2)$ & $17(54.8)$ & \\
\hline Female & $10(58.8)$ & $7(41.2)$ & \\
\hline Age (years) & & & 0.755 \\
\hline$<60$ & $17(51.5)$ & $16(48.5)$ & \\
\hline$\geq 60$ & $7(46.7)$ & $8(53.3)$ & \\
\hline $\mathrm{T}$ classification & & & 0.039 \\
\hline $\mathrm{T} 1 / \mathrm{T} 2$ & $17(65.4)$ & $9(34.6)$ & \\
\hline T3/T4 & $7(31.8)$ & $15(68.2)$ & \\
\hline Lymph node metastasis & & & 0.020 \\
\hline Positive & $7(30.4)$ & $16(69.6)$ & \\
\hline Negative & $17(68.0)$ & $8(32.0)$ & \\
\hline Pathological differentiation & & & 0.037 \\
\hline Well & $11(78.6)$ & $3(21.4)$ & \\
\hline Moderate & $7(41.2)$ & $10(58.8)$ & \\
\hline Poor & $6(35.3)$ & $11(64.7)$ & \\
\hline Clinical stage & & & 0.020 \\
\hline $\mathrm{I}+\mathrm{II}$ & $15(68.2)$ & $7(31.8)$ & \\
\hline III+IV & $9(34.6)$ & $17(65.4)$ & \\
\hline
\end{tabular}

Data were analysed using $\chi^{2}$ test.

Table II. Association between Wnt7a protein expression and clinicopathological parameters in patients with TSCC ( $\mathrm{n}=61$ ).

\begin{tabular}{|c|c|c|c|}
\hline \multirow[b]{2}{*}{ Clinicopathological parameters } & \multicolumn{2}{|c|}{ Wnt7a protein } & \multirow[b]{2}{*}{ P-value } \\
\hline & Low $(n=27)(\%)$ & High $(n=34)(\%)$ & \\
\hline Gender & & & 0.288 \\
\hline Male & $13(38.2)$ & $21(61.8)$ & \\
\hline Female & $14(51.9)$ & $13(48.1)$ & \\
\hline Age (years) & & & 0.134 \\
\hline$<60$ & $24(49.0)$ & $25(51.0)$ & \\
\hline$\geq 60$ & $3(25.0)$ & $9(75.0)$ & \\
\hline $\mathrm{T}$ classification & & & 0.033 \\
\hline $\mathrm{T} 1 / \mathrm{T} 2$ & $20(55.6)$ & $16(44.4)$ & \\
\hline $\mathrm{T} 3 / \mathrm{T} 4$ & $7(28.0)$ & $18(72.0)$ & \\
\hline Lymph node metastasis & & & 0.001 \\
\hline Positive & $8(24.2)$ & $25(75.8)$ & \\
\hline Negative & $19(67.9)$ & $9(32.1)$ & \\
\hline Pathological differentiation & & & $<0.0001$ \\
\hline Well & $15(88.2)$ & $2(11.8)$ & \\
\hline Moderate & $8(28.6)$ & $20(71.4)$ & \\
\hline Poor & $4(25.0)$ & $12(75.0)$ & \\
\hline Clinical stage & & & 0.002 \\
\hline $\mathrm{I}+\mathrm{II}$ & $16(69.6)$ & $7(30.4)$ & \\
\hline III+IV & $11(28.9)$ & $27(71.1)$ & \\
\hline
\end{tabular}

Data were analysed using $\chi^{2}$ test. 


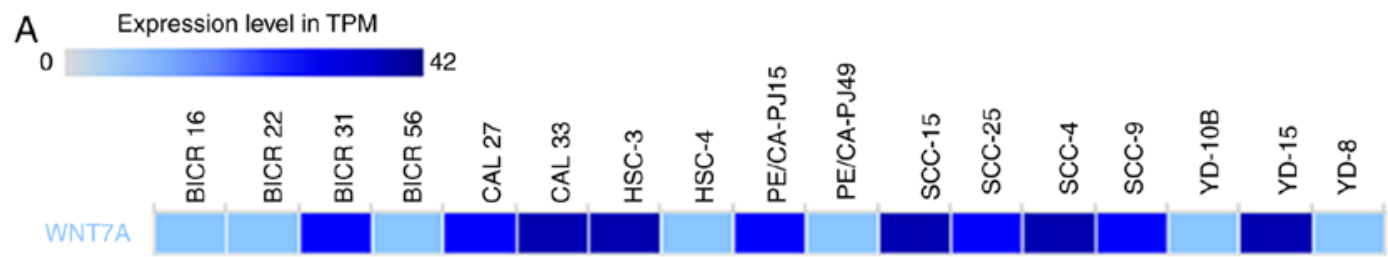

B

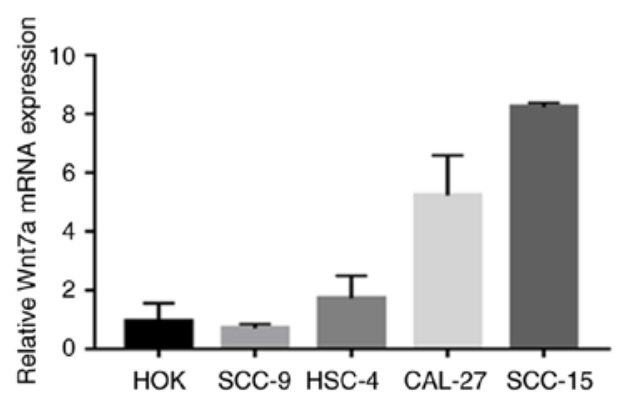

D

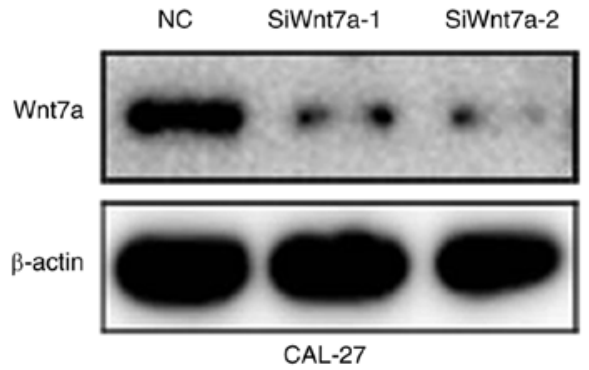

C
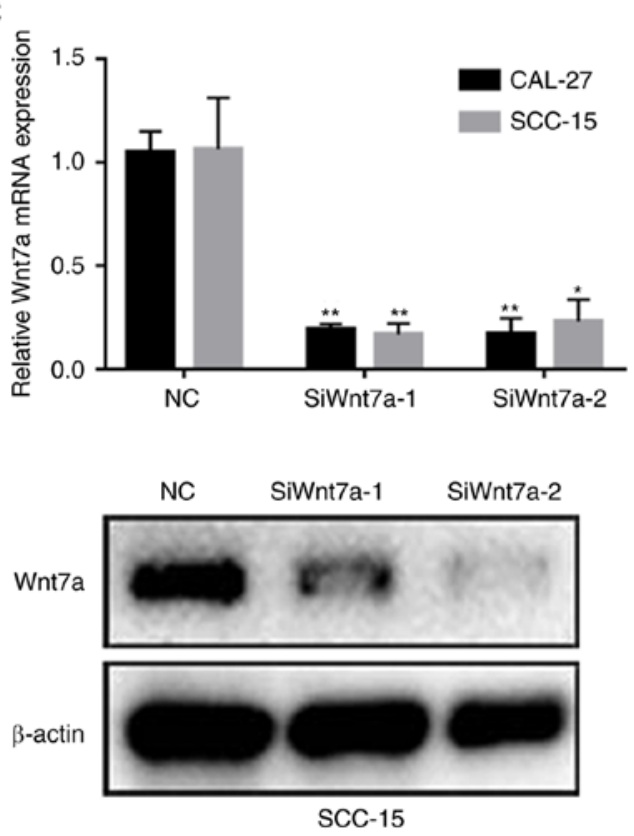

Figure 2. Expression of Wnt7a mRNA in TSCC cell lines and construction of Wnt7a-knockdown TSCC cell models. (A) Wnt7a mRNA expression in 17 TSCC cell lines from bioGPS. (B) Wnt7a mRNA expression in four TSCC cell lines, normalized to HOK, as detected by RT-qPCR. Wnt7a was efficiently downregulated following transfection of CAL-27 and SCC-15 cells with siRNA, as validated by (C) RT-qPCR (one-way analysis of variance and least significant difference test) and (D) western blotting. ${ }^{*} \mathrm{P}<0.05,{ }^{* *} \mathrm{P}<0.01$. HOK, human oral keratinocytes; NC, negative control; RT-qPCR, reverse transcription-quantitative polymerase chain reaction; $\mathrm{Si} /$ siRNA, small interfering RNA.

higher in tissues obtained from patients with lymph node metastasis (Fig. 1E), poor differentiation (Fig. 1F) and advanced TSCC (Fig. 1G). Wnt7a mRNA expression was also higher in tissues obtained from patients with lymph node metastasis (Fig. 1E) and advanced TSCC (Fig. 1G); however, it was not significantly associated with differentiation $(\mathrm{P}=0.087$; Fig. $1 \mathrm{~F})$. Furthermore, patients with higher Wnt7a mRNA (median recurrence-free survival, 1,366 days vs. 681 days, $\mathrm{P}=0.039$ ) and protein (median recurrence-free survival, 1,305 days vs. 469 days, $\mathrm{P}=0.024)$ expression had a shorter recurrence-free survival (Fig. 1H).

Construction of Wnt7a-knockdown TSCC cell models. Since high Wnt7a expression was associated with malignant phenotypes in patients with TSCC, the present study aimed to investigate the effects of Wnt7a on TSCC carcinogenesis and progression. BioGPS (http://biogps.org/\#goto=welcome) was used to observe Wnt7a mRNA expression in TSCC cell lines. A total of 10/17 TSCC cell lines recorded in the BioGPS exhibited elevated Wnt7a mRNA expression levels (Fig. 2A). The present study validated its expression in five cell lines using RT-qPCR; the results revealed that CAL-27 and SCC-15 cells exhibited the highest Wnt7a mRNA expression levels (Fig. 2B). To construct Wnt7a-knockdown TSCC cell models, two specific siRNAs targeting Wnt7a were transfected into CAL-27 and SCC-15 cell lines. Wnt7a siRNA transfection effectively knocked down the mRNA and protein expression of Wnt7a in CAL-27 and SCC-15 cells, as verified by RT-qPCR and western blotting, respectively (Fig. 2C and D).

Knockdown of Wnt7a suppresses proliferation of TSCC cells in vitro and in vivo. To detect the effects of Wnt7a on cell proliferation in TSCC cell lines, a CCK- 8 assay was performed. In addition, a tumorigenicity assay was performed in nude mice following implantation of Wnt7a-knockdown cells. As shown in Fig. 3A, cells transfected with siRNAs against Wnt7a exhibited reduced growth compared with in the NC group, thus indicating that the knockdown of Wnt7a may significantly suppress the proliferative ability in CAL-27 and SCC-15 cells. In addition, xenograft models were successfully constructed in BALB/c nude mice, using stable Wnt7a-knockdown CAL-27 cells (Fig. 3B). The results revealed that tumor volumes in the shRNA-Wnt7a group were markedly smaller than in the NC group (Fig. 3C). The tumor growth curves also revealed that knockdown of Wnt7a substantially inhibited tumor growth (Fig. 3D). These results indicated that Wnt7a knockdown suppressed the proliferation of TSCC cells.

Knockdown of Wnt7a suppresses migration and invasion of TSCC cells in vitro. Since Wnt7a expression was positively 
A

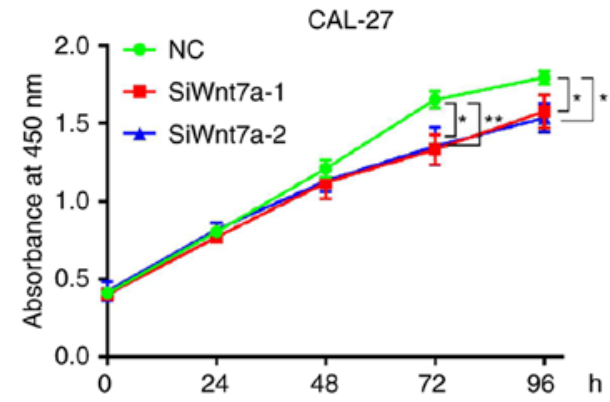

B

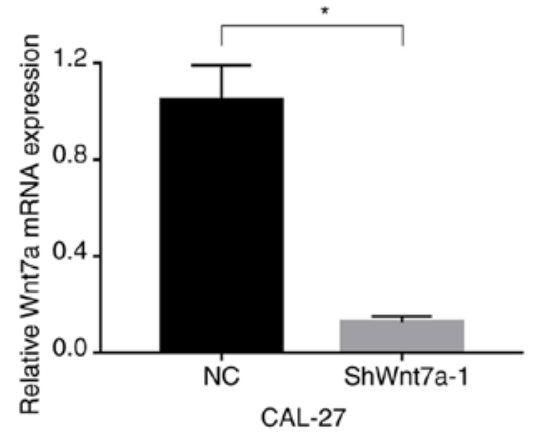

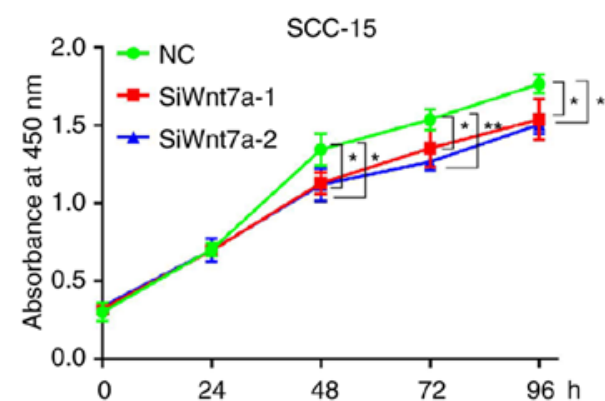

NC

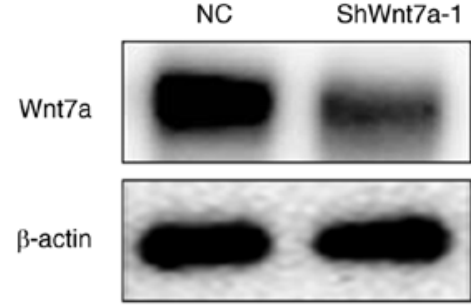

C

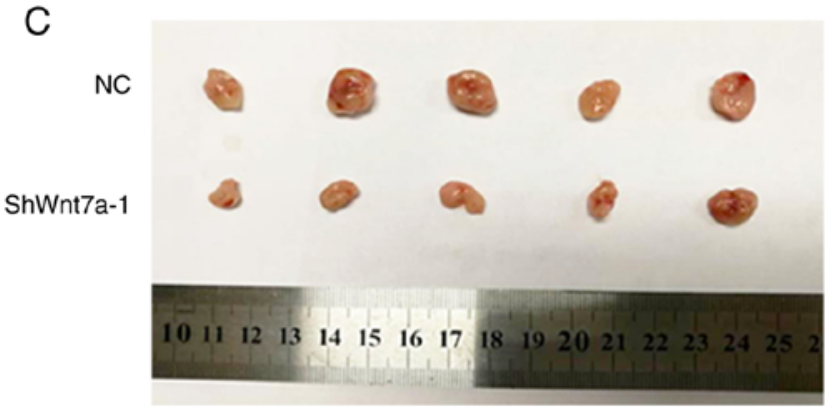

D

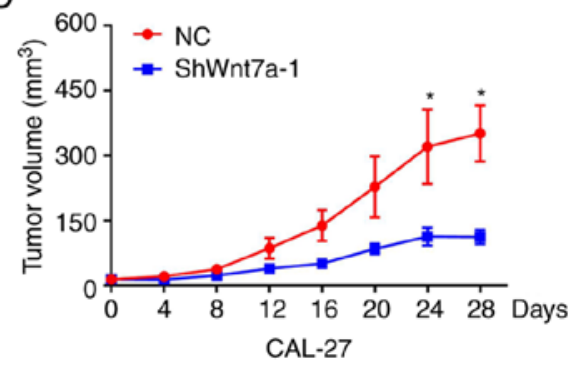

Figure 3. Effects of Wnt7a on proliferation in vitro and in vivo. (A) Transient knockdown of Wnt7a suppressed the proliferative ability of CAL-27 and SCC-15 cells, as measured by Cell Counting Kit- 8 assay (one-way analysis of variance and least significant difference test). (B) Stable lentivirus-mediated knockdown of Wnt7a in CAL-27 cells; the efficiency of Wnt7a knockdown was validated by reverse transcription-quantitative polymerase chain reaction (Student's t-test) and western blotting. (C) Tumorigenicity assay in nude mice revealed that tumor volume in the ShWnt7a-1 group was smaller than in the NC group. (D) Tumor growth curves revealed a significant difference between the ShWnt7a-1 group and the NC group (Student's t-test). ${ }^{*}<<0.05,{ }^{* *} \mathrm{P}<0.01$. NC, negative control; $\mathrm{Si}$, small interfering RNA; Sh, short hairpin RNA.

associated with lymph node metastasis, the present study further assessed the effects of Wnt7a on migration and invasion. Following Wnt7a knockdown, the numbers of cells that migrated $(\mathrm{P}<0.001$; Fig. $4 \mathrm{~A})$ and invaded $(\mathrm{P}<0.001$; Fig. 4B) through the membrane were significantly decreased in the CAL-27 and SCC-15 cell lines. These results revealed a functional role for Wnt7a in TSCC metastasis.

Knockdown of Wnt7a suppresses EMT in TSCC cells. Wnt7a modulates EMT and extracellular matrix degradation by targeting the canonical $\mathrm{Wnt} / \beta$-catenin signaling in bladder cancer (25). In the present study, western blotting was performed to investigate the expression of EMT markers in Wnt7a-knockdown TSCC cell lines. Silencing Wnt7a in CAL-27 and SCC-15 cells upregulated the epithelial marker, E-cadherin, whereas the expression levels of the mesenchymal markers, $\mathrm{N}$-cadherin, $\beta$-catenin and Vimentin, were inhibited, as was the EMT-associated transcriptional factor Snail (Fig. 5). These results indicated that Wnt7a may promote migration and invasion in TSCC by activating EMT activity.

\section{Discussion}

In most cases, diagnosis of TSCC is made when the disease has already progressed to the advanced stage. Invasion and metastasis are fundamental characteristics of advanced TSCC, and are the principal reasons behind the unfavorable outcomes for patients with TSCC. Numerous studies have suggested that Wnt signaling is associated with the metastasis and progression of TSCC (26-30); however, to the best of our knowledge, the specific ligand partners associated with Wnt signaling in this disease have not been identified. The present study revealed that Wnt7a was upregulated in TSCC tissues compared with in the corresponding adjacent non-cancerous tissues from two patient cohorts. Wnt7a expression was also revealed to be closely associated with tumor growth, lymph node metastasis and tumor staging. These results were concordant with those of a previous study that conducted gene expression profiling on advanced TSCC tissues, further supporting the idea that Wnt7a is frequently overexpressed in TSCC in the favorable prognostic predicted group (31). However, an opposite result was revealed in the present survival analysis; elevated Wnt7a 
A
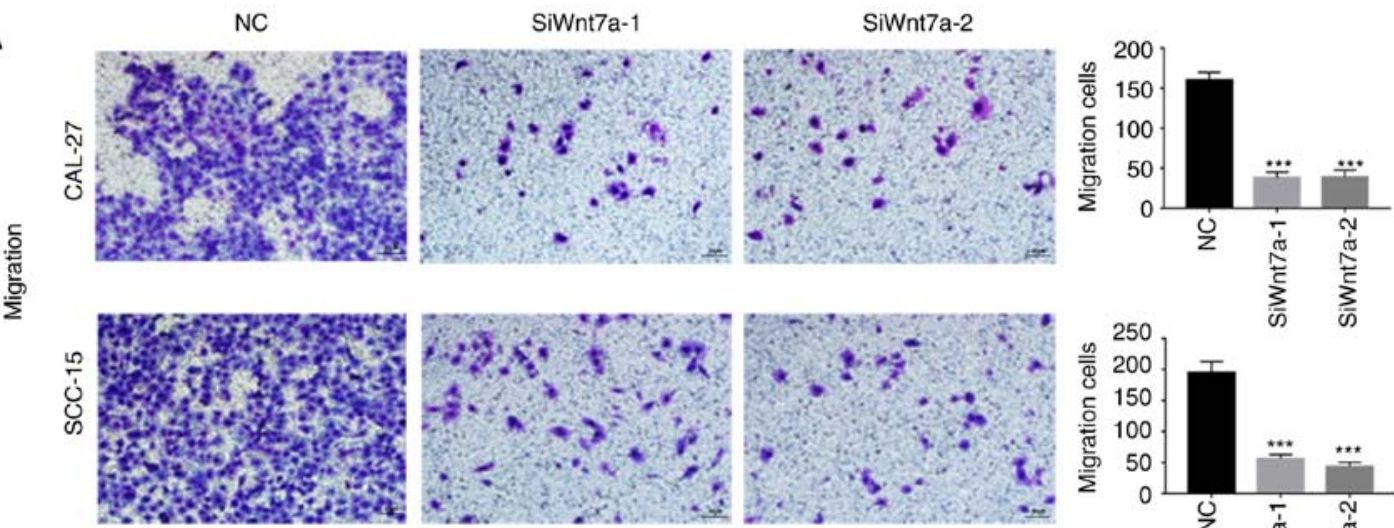

B
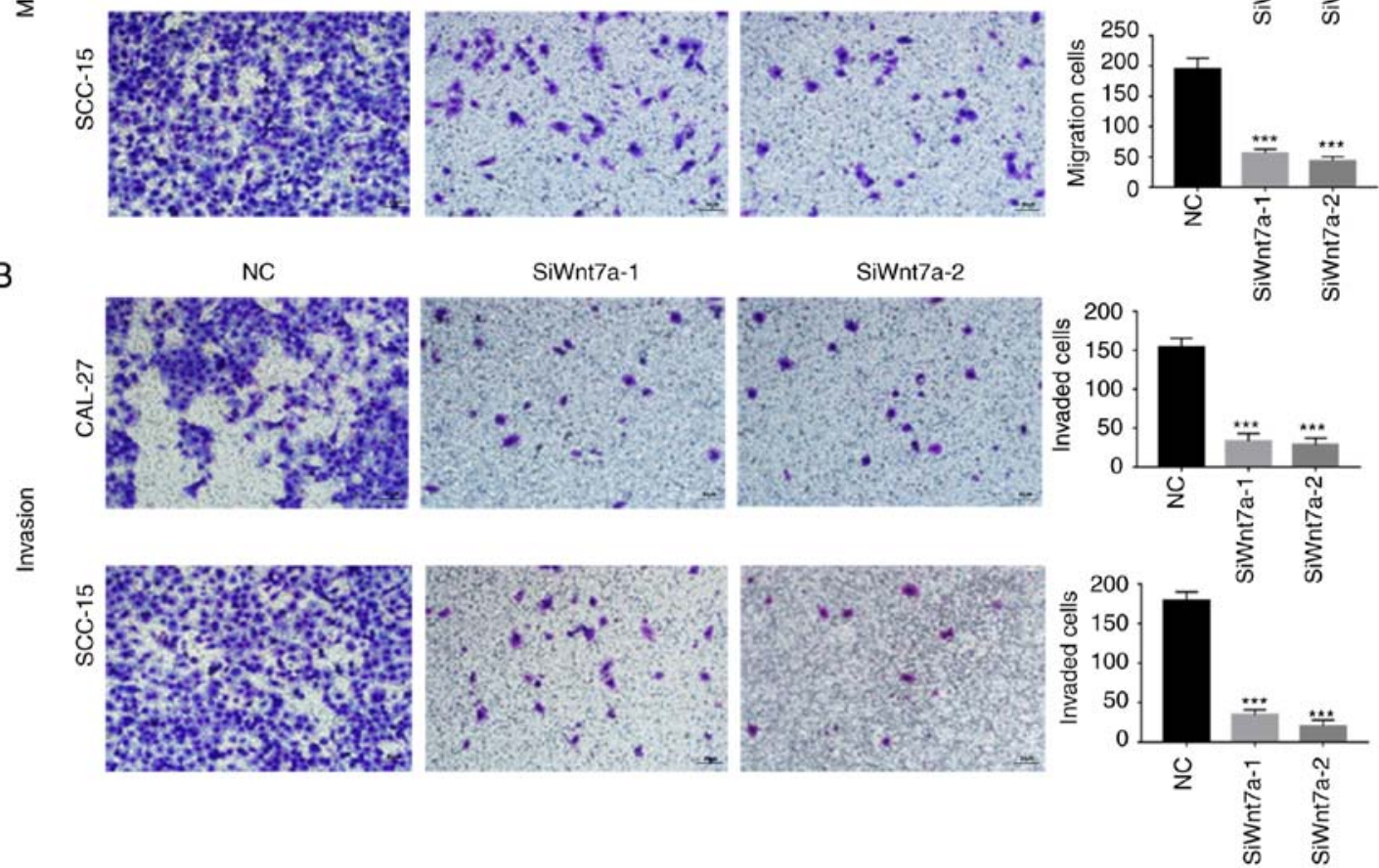

Figure 4. Effects of Wnt7a on the migration and invasion of CAL-27 and SCC-15 cells. (A) Transient knockdown of Wnt7a significantly inhibited the migratory ability of CAL-27 and SCC-15 cells, as determined by Transwell assays (one-way ANOVA and LSD test). (B) Transient knockdown of Wnt7a significantly inhibited the invasive ability of CAL-27 and SCC-15 cells, as determined by Transwell assays (one-way ANOVA and LSD test). ${ }^{* * *} \mathrm{P}<0.001$. Magnification, $\mathrm{x} 200$, scale bars $=50 \mu \mathrm{m}$. ANOVA, analysis of variance; LSD, least significant difference; NC, negative control; Si, small interfering RNA.
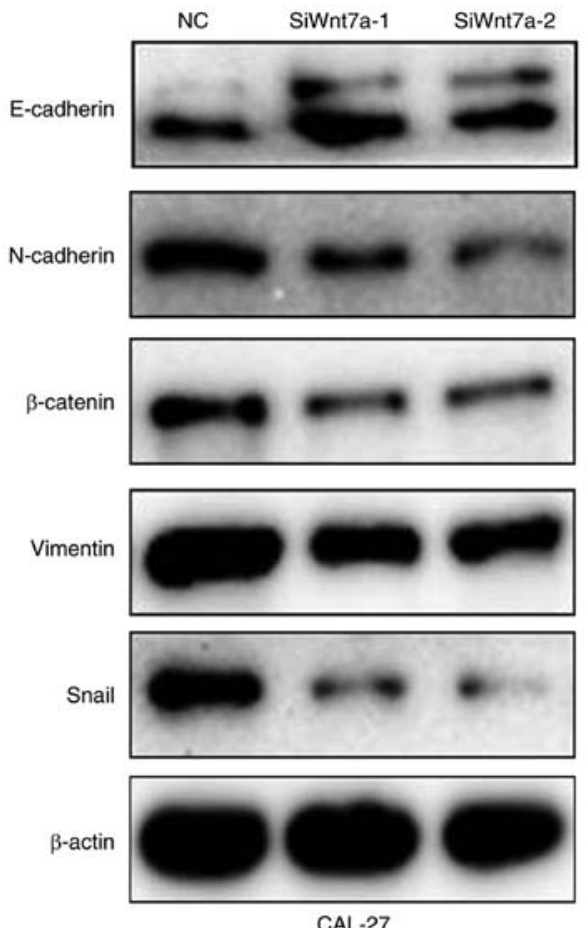
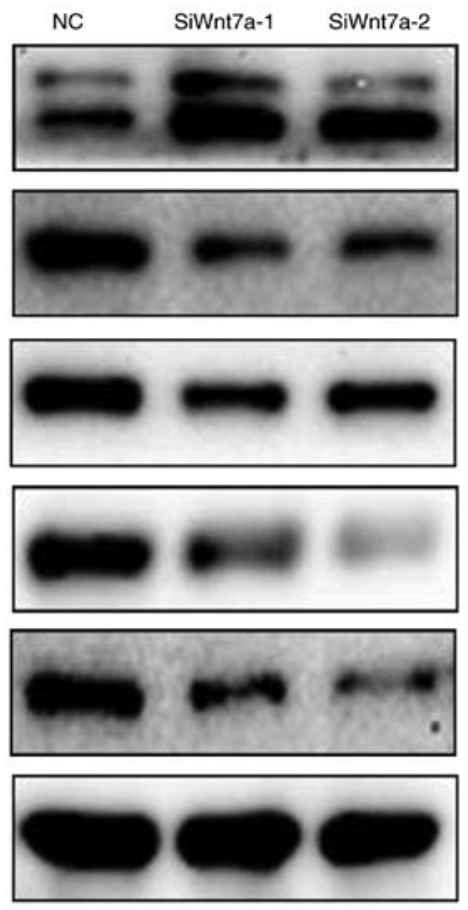

SCC-15

Figure 5. Effects of Wnt7a on EMT in CAL-27 and SCC-15 cells. Knockdown of Wnt7a upregulated the expression of the epithelial marker, E-cadherin, inhibited the mesenchymal markers, N-cadherin, $\beta$-catenin and Vimentin, as well as the EMT-associated transcriptional factor Snail, in CAL-27 and SCC-15 cells. EMT, epithelial-mesenchymal transition; NC, negative control; Si, small interfering RNA. 
expression was detected in patients with TSCC and an unfavorable prognosis. The results of the in vitro study revealed the pathological and tumorigenic roles of Wnt7a in regulating invasion and metastasis of TSCC. Knockdown of Wnt7a suppressed proliferation, migration and invasion, and reversed the EMT phenotype in TSCC cells. Therefore, the present study suggested that Wnt7a may act as an oncogene in TSCC and should be considered a candidate prognostic marker for patients with TSCC.

Local lymph node and distant organ metastases are believed to be critical features of TSCC, which contribute toward poor prognosis and patient mortality. Several biological behaviors are involved in this process, including EMT (32-35). EMT is an event whereby cancer cells lose their epithelial characteristics to acquire a mesenchymal phenotype, thereby enhancing their ability to migrate and invade, which is closely associated with cancer metastasis. Furthermore, EMT in cancer is associated with abnormal activation of several signaling pathways, including TGF- $\beta$, Wnt, epidermal growth factor and fibroblast growth factor $(34,36)$. Wnt/ $\beta$-catenin signaling has a major impact on EMT; however, the specific Wnt ligand and the underlying downstream mechanisms are not completely understood $(37,38)$.

The present study aimed to elucidate the role of $\mathrm{Wnt7a}$ in TSCC progression and EMT based on several reasons. Overexpression of Wnt7a has been reported in numerous malignant tumors, including endometrial cancer $(39,40)$, ovarian cancer $(18,41,23)$, colorectal cancer and pancreatic cancer (20); however, to the best of our knowledge, its role in oral cancer has not been examined. Wnt7a is mainly expressed in epithelial cells and has the capacity to control cell growth and maintain regular functioning of female reproductive organs (42-44). A previous study revealed that overexpression of Wnt7a in an ovarian cancer cell line results in the enhancement of its migratory and invasive capacities (45). In addition, it has been reported that Wnt7a, interacting with the $\mathrm{Wnt} / \beta$-catenin pathway, accelerates the growth and progression of ovarian cancer (18). Therefore, it may be hypothesized that Wnt7a is mechanically involved in the reprogramming of EMT.

Using RNA interference-mediated depletion of Wnt7a to investigate the biological function of Wnt7a in TSCC, it was revealed that Wnt7a was required for the proliferation, migration and invasion of CAL-27 and SCC-15 TSCC cells. Subsequently, Wnt7a knockdown in TSCC cells was demonstrated to lead to an acquisition of an epithelial phenotype, by enhancing the expression of E-cadherin, and a loss of the mesenchymal phenotype, by suppressing the expression of N-cadherin, Vimentin and the EMT-associated transcription factor, Snail. In addition, silencing Wnt7a suppressed the expression of $\beta$-catenin, thus indicating that Wnt7a regulated EMT by partially antagonizing the canonical Wnt/ $\beta$-catenin pathway. Therefore, it may be hypothesized that Wnt7a expression contributes to cancer aggressiveness by enhancing cell proliferation and metastasis, and accelerating the EMT process of TSCC.

In conclusion, the present results indicated that elevated expression of Wnt7a frequently occurred in TSCC tissues, and could be used to characterize tumor progression and predict its earlier recurrence. The present study confirmed that Wnt7a was involved in regulating cell proliferation, migration and invasion of TSCC cells, and established an important functional role for Wnt7a in TSCC pathogenesis, as revealed by its engagement in the EMT machinery.

However, the present study has several limitations. Firstly, the conclusions were based on the examination of two cell lines in which Wnt7a was silenced, and may not reflect the oncogenic role of Wnt7a in TSCC. A rescue experiment needs to be performed in the future to confirm that the re-expression of Wnt7a can restore tumor growth and metastasis of TSCC cells. Secondly, since this study was conducted under a retrospective design, further prospective studies with eligibility criteria applicable to clinical trials are required to confirm the findings that Wnt7a could accurately predict outcomes for patients with TSCC.

\section{Acknowledgements}

Not applicable.

\section{Funding}

The present study was financially supported by the Science and Technology Project of Guangzhou Province (201802020018) and the Medical Science and Technology Research Project of Guangdong (B2017103).

\section{Availability of data and materials}

The datasets used and/or analyzed during the current study are available from the corresponding author on reasonable request.

\section{Authors' contributions}

$\mathrm{XQ}$ and $\mathrm{JZ}$ were involved in the experimental conception and design. XQ and BJ performed the experiments. HC conducted the data analysis. XS contributed to the reagents, materials and tools. SX and XZ contributed to the conception of the study and wrote the manuscript. All authors read and approved the final manuscript. All authors contributed toward data analysis, drafting and critically revising the paper, and agree to be accountable for all aspects of the work.

\section{Ethics approval and consent to participate}

All patients provided informed consent for the use of their tissues and clinical data. The present study was approved by the Independent Ethics Committee of the Stomatological Hospital, Southern Medical University (approval no. [2018]05). The animal study was conducted in accordance with the Institutional Animal Care and Use Committee guidelines, and was approved by the Experimental Animal Ethics Committee of Southern Medical University (approval no. L2016113).

\section{Patient consent for publication}

All patients recruited provided consent for publication.

\section{Competing interests}

The authors declare that they have no competing interests. 


\section{References}

1. Magrath I and Litvak J: Cancer in developing countries: Opportunity and challenge. J Natl Cancer Inst 85: 862-874, 1993

2. Yuen AP, Lam KY, Chan AC, Wei WI, Lam LK, Ho WK and Ho CM: Clinicopathological analysis of elective neck dissection for N0 neck of early oral tongue carcinoma. Am J Surg 177: 90-92, 1999

3. Siegel R, Naishadham D and Jemal A: Cancer statistics, 2013. CA Cancer J Clin 63: 11-30, 2013.

4. Layland MK, Sessions DG and Lenox J: The influence of lymph node metastasis in the treatment of squamous cell carcinoma of the oral cavity, oropharynx, larynx, and hypopharynx: N0 versus N+. Laryngoscope 115: 629-639, 2005.

5. Bilancio A, Bontempo P, Di Donato M, Conte M, Giovannelli P, Altucci L, Migliaccio A and Castoria G: Bisphenol A induces cell cycle arrest in primary and prostate cancer cells through EGFR/ERK/p53 signaling pathway activation. Oncotarget 8 : $115620-115631,2017$.

6. Tripathi $\mathrm{K}$ and Garg $\mathrm{M}$ : Mechanistic regulation of epithelial-to-mesenchymal transition through RAS signaling pathway and therapeutic implications in human cancer. J Cell Commun Signal 12: 513-527, 2018.

7. Yang Z, Wang J, Pan Z and Zhang Y: miR-143-3p regulates cell proliferation and apoptosis by targeting IGF1R and IGFBP5 and regulating the Ras/p38 MAPK signaling pathway in rheumatoid arthritis. Exp Ther Med 15: 3781-3790, 2018.

8. Song K, Zheng G and Zhao Y: Liver kinase B1 suppresses growth of lung cancer cells through sonic hedgehog signaling pathway. Cell Biol Int 42: 994-1005, 2018

9. Fracalossi AC, Silva MS, Oshima CT and Ribeiro DA: Wnt/beta-catenin signalling pathway following rat tongue carcinogenesis induced by 4-nitroquinoline 1-oxide. Exp Mol Pathol 88: 176-183, 2010.

10. Bondos S: Variations on a theme: Hox and Wnt combinatorial regulation during animal development. Sci Stke 2006: pe38, 2006

11. Cadigan KM and Nusse R: Wnt signaling: A common theme in animal development. Genes Dev 11: 3286-3305, 1997.

12. Jiao J, Zhao X, Liang Y, Tang D and Pan C: FGF1-FGFR1 axis promotes tongue squamous cell carcinoma (TSCC) metastasis through epithelial-mesenchymal transition (EMT). Biochem Biophys Res Commun 466: 327-332, 2015.

13. Zhang H, Sun JD, Yan LJ and Zhao XP: PDGF-D/PDGFRbeta promotes tongue squamous carcinoma cell (TSCC) progression via activating p38/AKT/ERK/EMT signal pathway. Biochem Biophys Res Commun 478: 845-851, 2016.

14. Liu L, Wu B, Cai H, Li D, Ma Y, Zhu X, Lv Z, Fan Y and Zhang $\mathrm{X}$ : Tiam 1 promotes thyroid carcinoma metastasis by modulating EMT via Wnt/ $\beta$-catenin signaling. Exp Cell Res 362 532-540, 2018

15. Cui D, Zhao $\mathrm{Y}$ and Xu J: Activated CXCL5-CXCR2 axis promotes the migration, invasion and EMT of papillary thyroid carcinoma cells via modulation of beta-catenin pathway. Biochimie 148: 1-11, 2018.

16. Ghahhari NM and Babashah S: Interplay between microRNAs and WNT/beta-catenin signalling pathway regulates epithelial-mesenchymal transition in cancer. Eur J Cancer 51: 1638-1649, 2015

17. Nie X, Liu Y, Chen WD and Wang YD: Interplay of miRNAs and canonical wnt signaling pathway in hepatocellular carcinoma. Front Pharmacol 9: 657, 2018.

18. Yoshioka S, King ML, Ran S, Okuda H, MacLean JA II, McAsey ME, Sugino N, Brard L, Watabe K and Hayashi K: WNT7A regulates tumor growth and progression in ovarian cancer through the WNT/ $\beta$-catenin pathway. Mol Cancer Res 10: 469-482, 2012.

19. Avgustinova A, Iravani M, Robertson D, Fearns A, Gao Q, Klingbeil P, Hanby AM, Speirs V, Sahai E, Calvo F and Isacke CM: Tumour cell-derived Wnt7a recruits and activates fibroblasts to promote tumour aggressiveness. Nat Commun 7: 10305, 2016.

20. Kirikoshi $\mathrm{H}$ and Katoh $\mathrm{M}$ : Expression of $W N T 7 A$ in human normal tissues and cancer, and regulation of $W N T 7 A$ and $W N T 7 B$ in human cancer. Int J Oncol 21: 895-900, 2002.

21. Bikkavilli RK, Avasarala S, Van Scoyk M, Arcaroli J, Brzezinski C, Zhang W, Edwards MG, Rathinam MK, Zhou T, Tauler J, et al: Wnt7a is a novel inducer of $\beta$-catenin-independent tumor-suppressive cellular senescence in lung cancer. Oncogene 34: 5406, 2015.

22. Sato N, Fukushima N, Maitra A, Matsubayashi H, Yeo CJ, Cameron JL, Hruban RH and Goggins M: Discovery of novel targets for aberrant methylation in pancreatic carcinoma using high-throughput microarrays. Cancer Res 63: 3735-3742, 2003.
23. Zhang XL, Peng CJ, Peng J, Jiang LY, Ning XM and Zheng JH: Prognostic role of Wnt7a expression in ovarian carcinoma patients. Neoplasma 57: 545-551, 2010.

24. Livak KJ and Schmittgen TD: Analysis of relative gene expression data using real-time quantitative PCR and the $2^{-\Delta \Delta C_{\mathrm{T}}}$ method. Methods 25: 402-408, 2001.

25. Huang X, Zhu H, Gao Z, Li J, Zhuang J, Dong Y, Shen B, Li M, Zhou H, Guo H, et al: Wnt7a activates canonical Wnt signaling, promotes bladder cancer cell invasion, and is suppressed by miR-370-3p. J Biol Chem 293: 6693-6706, 2018.

26. Paluszczak J, Kiwerska K, Mielcarek-Kuchta D: Frequent methylation of $D A B 2$, a Wnt pathway antagonist, in oral and oropharyngeal squamous cell carcinomas. Pathol Res Pract 214: 314-317, 2018.

27. Hema KN, Smitha T, Sheethal HS and Mirnalini SA: Epigenetics in oral squamous cell carcinoma. J Oral Maxillofac Pathol 21: 252-259, 2017.

28. Farooqi AA, Shu CW, Huang HW, Wang HR, Chang YT, Fayyaz S, Yuan SF, Tang JY and Chang HW: TRAIL, Wnt, Sonic Hedgehog, TGF $\beta$, and miRNA signalings are potential targets for oral cancer therapy. Int J Mol Sci 18: pii: E1523, 2017.

29. Gonzalez-Moles MA, Ruiz-Avila I, Gil-Montoya JA, Plaza-Campillo J and Scully C: $\beta$-catenin in oral cancer: An update on current knowledge. Oral Oncol 50: 818-824, 2014.

30. Noguti J, DE Moura CF, Hossaka TA, Franco M, Oshima CT, Dedivitis RA and Ribeiro DA: The role of canonical WNT signaling pathway in oral carcinogenesis: A comprehensive review. Anticancer Res 32: 873-878, 2012.

31. Enokida T, Fujii S, Takahashi M, Higuchi Y, Nomura S, Wakasugi T, Yamazaki T, Hayashi R, Ohtsu A and Tahara M: Gene expression profiling to predict recurrence of advanced squamous cell carcinoma of the tongue: Discovery and external validation. Oncotarget 8: 61786-61799, 2017.

32. Shetty AV and Wong DJ: Systemic treatment for squamous cell carcinoma of the head and neck. Otolaryngol Clin North Am 50: 775-782, 2017.

33. Almangush A, Heikkinen I, Makitie AA, Coletta RD, Läärä E, Leivo I and Salo T: Prognostic biomarkers for oral tongue squamous cell carcinoma: A systematic review and metaanalysis. Br J Cancer 117: 856-866, 2017.

34. Brabletz T, Kalluri R, Nieto MA and Weinberg RA: EMT in cancer. Nat Rev CanceR 18: 128-134, 2018.

35. Vig N, Mackenzie IC and Biddle A: Phenotypic plasticity and epithelial-to-mesenchymal transition in the behaviour and therapeutic response of oral squamous cell carcinoma. J Oral Pathol Med 44: 649-655, 2015.

36. Lee JM, Dedhar S, Kalluri R and Thompson EW: The epithelial-mesenchymal transition: New insights in signaling, development, and disease. J Cell Biol 172: 973-981, 2006.

37. Thiery JP, Acloque H, Huang RY and Nieto MA: Epithelialmesenchymal transitions in development and disease. Cell 139: 871-890, 2009.

38. Vincan $\mathrm{E}$ and Barker $\mathrm{N}$ : The upstream components of the Wnt signalling pathway in the dynamic EMT and MET associated with colorectal cancer progression. Clin Exp Metastasis 25: 657-663, 2008.

39. Liu Y, Meng F, Xu Y, Yang S, Xiao M, Chen X and Lou G: Overexpression of Wnt7a is associated with tumor progression and unfavorable prognosis in endometrial cancer. Int J Gynecol Cancer 23: 304-311, 2013.

40. Peng C, Zhang X, Wang Y, Li L, Wang Q and Zheng J: Expression and prognostic significance of wnt $7 \mathrm{a}$ in human endometrial carcinoma. Obstet Gynecol Int 2012: 134962, 2012.

41. King ML, Lindberg ME, Stodden GR, Okuda H, Ebers SD, Johnson A, Montag A, Lengyel E, MacLean Ii JA and Hayashi K: WNT7A/ $\beta$-catenin signaling induces FGF1 and influences sensitivity to niclosamide in ovarian cancer. Oncogene 34: 3452-3462, 2015.

42. Tulac S, Nayak NR, Kao LC, Van Waes M, Huang J, Lobo S, Germeyer A, Lessey BA, Taylor RN, Suchanek E, et al: Identification, characterization, and regulation of the canonical Wnt signaling pathway in human endometrium. J Clin Endocrinol Metab 88: 3860-3866, 2003.

43. Miller C and Sassoon DA: Wnt-7a maintains appropriate uterine patterning during the development of the mouse female reproductive tract. Development 125: 3201-3211, 1998.

44. Hayashi K, Erikson DW, Tilford SA, Bany BM, Maclean JA II, Rucker EB III, Johnson GA and Spencer TE: Wnt genes in the mouse uterus: Potential regulation of implantation. Biol Reprod 80: 989-1000, 2009.

45. Merritt MA, Parsons PG, Newton TR, Martyn AC, Webb PM, Green AC, Papadimos DJ and Boyle GM: Expression profiling identifies genes involved in neoplastic transformation of serous ovarian cancer. BMC Cancer 9: 378, 2009. 